\title{
Contenus et valeurs
}

Bigarrure du monde, convergence des questions

Content and values. Convergent questions in a diverse world

Contenidos y valores. Mezcolanza del mundo, convergencia de cuestiones

\section{Roger-François Gauthier et Florence Robine}

\section{OpenEdition}

\section{Journals}

Édition électronique

URL : https://journals.openedition.org/ries/744

DOI : $10.4000 /$ ries. 744

ISSN : 2261-4265

\section{Éditeur}

France Education international

Édition imprimée

Date de publication : 1 décembre 2009

Pagination : 77-84

ISBN : 978-2-8542-0577-0

ISSN : $1254-4590$

Référence électronique

Roger-François Gauthier et Florence Robine, "Contenus et valeurs », Revue internationale d'éducation de Sèvres [En ligne], 52 | décembre 2009, mis en ligne le 01 décembre 2012, consulté le 26 avril 2022. URL : http://journals.openedition.org/ries/744 ; DOI : https://doi.org/10.4000/ries.744 


\title{
Contenus et valeurs
}

\section{Bigarrure du monde, convergence des questions}

\author{
Roger-François Gauthier, \\ Florence Robine
}

Le thème des contenus d'enseignement et des valeurs diffusées par les écoles des différents systèmes éducatifs ne pouvait pas ne pas être traité à l'occasion de la question initiale du colloque : «Un seul monde, une seule école ? » ${ }^{1}$. Toutefois, pour incontournable qu'elle soit, la question des contenus et valeurs ne nous est pas apparue si banale : il ne s'est en effet pas agi, entre les différents interlocuteurs et notamment à partir des exposés proposés sur les situations anglaise, française, indienne, sénégalaise et suédoise, de s'intéresser à des questions de gestion des curricula, par exemple, mais de plonger un niveau au-dessous, dans les zones en général moins fréquentées, au plan international, des contenus euxmêmes et même, excursion plus rare encore, des valeurs qui les sous-tendent ${ }^{2}$.

Si l'on s'intéresse en général peu à cet objet dans les comparaisons internationales, c'est, sans doute, avec d'autres motifs, qu'on considère qu'il ne recouvre que des choses qu'il n'est guère pertinent de comparer, soit que les enseignements soient considérés a priori comme similaires, soit au contraire que les pays fondent sur leur souveraineté de développer des contenus propres, dont le rapprochement avec ceux d'autres pays n'a par construction plus guère de nécessité. Il nous est en fait apparu que ce thème nous engageait aussi bien à dégager de grandes problématiques communes à tous les pays abordés qu'à mettre en évidence les spécificités fondamentales de systèmes rapprochés : nous nous demanderons si précisément ce respect de la diversité associé à une préoccupation pour une école dispensant des contenus cohérents et responsables n'est pas un caractère commun des systèmes du village mondial de la fin de cette décennie.

\section{UNE QUESTION DÉSORMAIS VIVE... \\ L'école interpellée sur ce qu'elle enseigne}

Le premier constat semble devoir être celui de toutes les demandes qui désormais accourent vers l'école quant à la question de ce qu'elle enseigne : l'époque n'est plus où l'école, comme la religion jadis, imposait un contenu au

1. Cet article s'appuie sur les éléments recueillis par Valérie Tehio lors de l'atelier sur ce thème.

2. Le numéro 49 de la Revue internationale d'éducation de Sèvres ("Quel avenir pour les études en sciences humaines ? ", décembre 2008) s'intéresse à cette question des valeurs sous-jacentes. 
nom d'une transcendance ; aujourd'hui, l'école est confrontée à des attentes qu'un grand nombre d'instances placent en elle, et ces attentes portent notamment sur la question des contenus. Les systèmes d'éducation avaient rarement été constitués pour répondre à des attentes.

Elles viennent non seulement de membres des sociétés en question, par exemple de milieux professionnels ou économiques, de certains groupes sociaux ou idéologiques, mais aussi, selon les cas, de modes étrangères, d'organisations internationales (l'Union européenne et les compétences-clés par exemple) ou de bailleurs de fonds (dans le cas, par exemple, de pays en développement).

L'une des questions posées est de savoir quelle position les systèmes d'éducation peuvent adopter face à cette pluralité de demandes hétérogènes et renvoyant à des valeurs qui peuvent être disparates, ou confuses : entre une position de sanctuaire, marquant la fermeture à ce qui ne relève pas des canons scolaires et qui produit des contenus inadaptés, et une position d'ouverture à tous les vents qui produit l'incohérence, les systèmes essaient de trouver différents modes de gestion de la demande dans un cadre clair d'expertise et de décision politique.

\section{La diversité des contenus : une éminente valeur}

Si l'on descend plus profondément que l'apparence des programmes, qui ne se laissent pas toujours décrypter au premier regard, on s'aperçoit que les différents pays se réfèrent à des valeurs qui sont en fait largement différentes et qui expriment des éléments importants des différentes sociétés.

La Suède, par exemple, privilégie de toute évidence la valeur de démocratie, à la fois en veillant à ce que la structure générale du curriculum soit sanctionnée par un vote du Parlement, mais surtout en plaçant à l'entrée du curriculum même des mises en gardes et avertissements forts comme : «...La démocratie forme la base du système national d'éducation (...). L'école a pour mission de faire partager et de transmettre aux élèves ces valeurs fondamentales sur lesquelles notre société repose : l'inviolabilité de la personne humaine, la liberté et l'intégrité individuelle, la valeur égale de chacun, l'égalité entre les hommes et les femmes, la solidarité avec les plus faibles et les plus vulnérables sont autant de valeurs que l'école doit incarner et transmettre. »

L'Inde renvoie à une visée universelle ainsi qu'à des valeurs " séculières ", en insistant sur des concepts comme celui d'éducation à la paix : "Prenant ses distances avec les références religieuses et morales traditionnelles, la National Policy on Education a fondé son action sur les valeurs laïques inscrites dans la Constitution indienne et sur les principes qui ont inspiré le peuple indien dans sa lutte pour la liberté...». 
L'exemple anglais est encore plus caractéristique, puisqu'il est intéressant de voir aussi bien l'importance donnée dans les textes à l'ouverture prévenante à autrui ( care»), à la notion de développement individuel de chaque élèves ("Learning objectives should be right for each child at each stage of their learning »), au concept de multiculturalisme ou encore à l'objectif du plaisir d'apprendre ("to give teachers discretion to find the best ways to inspire in their pupils a joy and a commitment to learning »).

Dans la référence française, la spécificité des «programmes » est que ce qu'on enseigne à l'école est d'abord là pour fonder le contrat social et politique, en permettant aux hommes de "rejeter les erreurs dont on veut les rendre victimes " : éclairer par le savoir, pour faire des citoyens.

Ces différences donnent l'idée qu'existe, au-delà de tendances unificatrices et mondialisantes ou européennes, une très précieuse pédago-diversité, que les comparaisons internationales devraient nous faire mieux appréhender et qui doit être protégée et valorisée.

\section{UNE COHÉRENCE RECHERCHÉE DES CONTENUS ET DES VALEURS}

Si l'on est frappé de cette diversité, on ne l'est pas moins de la convergence de l'attitude de la plupart des responsables qui, dans tous les pays, portent un regard de plus en plus vigilant sur la qualité des contenus proposés. Pour les mêmes motifs qu'il était précédemment rappelé, les contenus ne " vont plus de soi ", pour les différents systèmes, mais sont au contraire l'objet de travaux pour chercher les meilleures réponses, aux plans politique, scientifique et pédagogique. Cette question est devenue une question de premier plan pour les systèmes. Cette vigilance vers la qualité des contenus a plusieurs dimensions :

\section{La recherche des cohérences}

Ce à quoi les systèmes semblent le plus attachés est d'abord d'assurer les cohérences internes à la prescription pédagogique, entre les différents niveaux d'enseignement - et la structure simple de l'école obligatoire en Suède est par exemple un atout considérable - ou entre les différentes disciplines. La recherche de la cohérence va bien au-delà, puisqu'on tend à s'assurer aussi que le contenu des programmes (au sens cognitif, les « syllabi ») soit corrélé aux valeurs à transmettre, aux «compétences » (et pas seulement contenus cognitifs) que les élèves doivent maîtriser, compétences plus ou moins disciplinaires, plus ou moins transversales. De même, la prescription des contenus semble incomplète si elle n'englobe pas tout ce qui relève de l'évaluation et des examens. Il existe donc une visée de plus en plus holistique des prescriptions, dans le cadre de curricula, 
et un pays jusqu'ici réfractaire à la mise en place de curricula comme la France a pourtant adopté, en 2006, un Socle commun qui est un premier pas vers une politique curriculaire cohérente.

\section{La volonté d'explicitation}

Un caractère commun à de nombreux projets curriculaires est celui du souci de l'explicitation claire de ce qui est prescrit, pour les professeurs, les familles et les élèves : c'est la conséquence d'observations faites depuis longtemps relativement à l'effet négatif qu'avait sur les élèves les plus fragiles l'écart entre le "curriculum formel » et le «curriculum caché ». Le sens caché du curriculum notamment en termes de valeurs sous-jacentes, est de plus en plus exposé, explicité.

\section{Des contenus associés à l'objectif général d'amélioration du système}

Beaucoup de systèmes ont faite leur l'idée selon laquelle assurer des contenus de qualité ne se limite pas à élaborer une prescription, fût-elle la meilleure du monde : il s'agit de faire de la qualité des contenus un élément relié à d'autres facteurs d'amélioration de l'école. La mise en ouvre du National Curriculum anglais l'illustre bien : il a été appliqué en même temps qu'étaient mises en place les évaluations nationales ainsi que le nouveau rôle de l'Ofsted ${ }^{3}$. Bien plus : l'idée se fait jour en certains pays que les contenus ne seraient jamais un objectif statique, mais un objet en perpétuelle "révision », dans le sens de « monter les standards» et de tirer les acquis des élèves vers le haut.

\section{L'attention portée à la mise en œuvre locale}

Dans beaucoup de pays, l'idée domine que les « contenus prescrits » ne prennent vraiment d'importance que quand ils se réalisent en contenus réels, dans le cadre de chaque école, et ils sont élaborés nationalement pour laisser précisément la place à des négociations et à une prise en mains locale, souvent sous une forme aussi explicite.

\section{La cohérence des interventions des acteurs}

Ce point a été relevé avec insistance : ne faut-il pas, si l'on veut assurer effectivement la jonction " contenus-valeurs » qu'on estime indispensable, veiller à ce que l'attitude-même des différents acteurs et notamment des enseignants soit conforme à cette association ? Les enseignants, s'interrogeait-on, ne doivent-ils pas tous enseigner aussi bien des valeurs que des matières»?

3. Office for Standards in Education. 


\section{La mise en place de procédures adaptées d'élaboration}

Le souci de la qualité du produit fini est souvent à l'origine de la recherche des précautions les plus adéquates : associer à l'élaboration toute la diversité d'experts requise (spécialistes de la discipline, spécialistes de pédagogie, professeurs en exercice etc.), préparer de la façon la plus responsable et si possible consensuelle une décision qui apparaît comme politique... Certains pays, comme la Suède, ont défini une véritable procédure où gouvernement et Parlement ont chacun un rôle défini. Cette recherche de transparence, de consensus est importante et si dans certains pays, comme la France, il n'existe pas de règle pérenne d'élaboration des contenus dont l'origine et le statut juridique sont divers, on voit ailleurs se constituer un quasi " droit des curricula » qui montre ce souci à la fois de démocratie et d'efficacité.

\section{Le besoin de vision}

Les meilleures procédures ne dispensent nulle part, sur un sujet comme celui-ci, les systèmes éducatifs d'avoir une «vision » sur l'école et les apprentissages des élèves. Quand cela n'existe pas, ou si elle n'est pas portée au niveau suffisant, ce que rappela l'intervention sénégalaise à propos de l'enseignement des sciences, on a peu de chances de réussir à mettre en place les contenus souhaités.

\section{Contradictions}

Mais si l'école s'est saisie des contenus et valeurs qu'elle enseigne avec la conscience de l'importance de ce qui se joue là, elle a toutefois dû faire immédiatement, et dans toutes les situations, le constat qu'une valeur est par définition ce qui n'existe pas et qu'il existe une tension majeure et quasi irréductible entre une institution qui «enseigne des valeurs » et le monde de l'Être, sous ses formes infinies.

D'où une question : jusqu'où l'école peut-elle se poser contre le monde et s'opposer à lui ? Chacun sera certes d'accord pour dire que l'école ne doit pas être une école de la haine sociale ou raciale, mais comment va-t-on traiter, par exemple, les conflits de valeurs qui ne manqueront pas de surgir entre des programmes qui mettent au premier plan une référence nationale, traditionnelle et identitaire, et des programmes privilégiant une référence centrale, plus ouverte, à l'humanité ? La fonction même de transmission, traditionnellement reconnue à l'école, demande d'être revisitée : faut-il tout " transmettre »? Quelle doit être la part entre la transmission et l'invention de nouveaux chemins ?

L'école doit-elle faire siens les jeux du monde économique et la « valeur » générale de concurrence qui est la sienne, ou doit-elle plutôt mettre en avant des valeurs de solidarité et d'égalité qu'elle risque bien d'être la seule à enseigner ? 
Doit-on considérer que sa fonction distanciée doit lui permettre précisément de présenter aux élèves la diversité, voire les conflits de valeurs? Sans doute, mais en faisant de la sorte, elle privilégie elle-même une valeur de distanciation et de débat argumenté qui n'est pas la sienne partout. Face à des élèves quotidiennement mitraillés par la propagande universelle et béate pour la loi souveraine du marché et les bienfaits du développement concurrentiel, ainsi que confrontés à l'immensité des ressources de l'Internet, l'école doit-elle, peut-elle, a-t-elle la force d'introduire d'autres propositions et un regard critique?

On voit à quel point tout cela est difficile et ne peut se résoudre que par un débat démocratique sur ce qu'on attend de l'école dans les différentes situations : la seule certitude est qu'on ne peut plus contourner ces questions. Et ce débat démocratique ne doit-il pas être, de plus en plus, conduit à l'échelle d'" un seul monde ", comme l'a stipulé l'acte constitutif de l'UNESCO : «les guerres prenant naissance dans l'esprit des hommes, c'est dans l'esprit des hommes que doivent être élevées les défenses de la paix " ?

Mais il y a plus directement préoccupant : il apparaît en effet que la question des tensions autour des valeurs est pour l'école encore plus délicate par le fait qu'elle se pose en son sein : la plupart des systèmes perçoivent qu'ils ne pourront se satisfaire de leurs travaux sur les contenus et les valeurs que l'école retient d'enseigner tant que des solutions n'auront pas été trouvées à toutes les situations où l'école même fait le contraire de ce qu'elle dit !

Cela va de l'écart entre la promesse démocratique, valeur s'il en est, de bien des systèmes scolaires et la réalité des inégalités sociales et culturelles que l'école ne parvient pas à compenser, quand elle ne les creuse pas, à la faiblesse de la réalité démocratique et solidaire de l'organisation de la classe et de la vie scolaire en bien des cas, à la préoccupation élitiste que de nombreux systèmes scolaires nourrissent en leur sein, ainsi qu'à l'obsession de la compétition dans laquelle ils installent souvent les enfants de façon excessive. Non seulement cet élitisme forcené prive la collectivité de cerveaux et de compétences qui seraient nécessaires, mais il est souvent absurde (on évalue, par exemple, parce que c'est plus simple et moins onéreux, à partir de la restitution mécanique de cours en contradiction avec un discours sur l'importance de la démarche expérimentale ou des compétences de communication), il est injuste par le fait que les conditions de l'enseignement sont inégalitaires (comme l'a montré la présentation sénégalaise), mais surtout il fabrique échec et humiliation. Les pratiques des classes de préparation des examens telles qu'elles sont évoquées dans de nombreux pays, à l'illustration de ce qui en a été dit pour l'Inde ou le Sénégal, montrent qu'on est en effet très loin du respect des valeurs.

Mais vouloir protéger les élèves contres les excès de l'esprit de compétition n'implique-t-il pas aussi de repenser la compétition dans laquelle les systèmes scolaires eux-mêmes se sont engagés, par des classements souvent 
discutables au plan de l'équité entre les écoles ou par les classements internationaux, opérés à partir de certains indicateurs, sans que le niveau de cohésion sociale créée par l'école, par exemple, ou celui du bonheur des élèves à s'y trouver soit toujours évalué ?

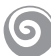

Que sait-on de l'effet des contenus et valeurs enseignés sur les destinataires de l'enseignement? On peut considérer que beaucoup d'écoles n'ont pas terminé le mouvement commencé par certaines, consistant à faire en sorte que les contenus et valeurs diffusés ne soient pas un objet absolu ni définitif, mais un ensemble de propositions à considérer avec le souci constant d'évaluer leur pertinence, leur mise en œuvre par les maîtres et particulièrement leur réception par les élèves... C'est particulièrement vrai pour l'enseignement des valeurs : tous ont dit leur désarroi pour tenter d'évaluer, et du coup de faire progresser, l'effet de l'école sur les élèves en matière d'enseignement de valeurs individuelles et collectives. La question aussi non pas de l'utilitarisme à court terme, mais de l'effet profond, cognitif, " compétentiel », culturel, social, comportemental, etc., de tel ou tel apprentissage, selon les catégories d'élèves, permettrait de faire des choix avec plus de clarté dans ce domaine encore largement constitué de certitudes acquises par la tradition. Des recherches internationales seraient à lancer sur ces thèmes.

Contenus et valeurs : l'école est la seule instance à poser cette question comme elle le fait, et il est indispensable qu'elle le fasse. Il semblerait que le temps soit venu pour que non seulement les responsables, mais aussi le public s'arrête à la question de savoir ce que sont les savoirs scolaires au XXI ${ }^{\mathrm{e}}$ siècle : certainement plus une vérité révélée par une religion ou par une science de type positiviste, mais, entre les mille savoirs, expertises et opinions qui clignotent et palpitent d'autant plus dans le monde que leur diffusion n'est pas et ne sera plus majoritairement demandée à l'école, une tentative unique, indispensable, équitable, prométhéenne, de proposer des synthèses destinées à aider les petits très inachevés de l'espèce humaine. Au dessus de l'erreur et de l'illusion, comme de l'éclatement des communautés dont ils relèvent peut-être, à aller vers un peu plus d'intelligibilité, de conscience et de maîtrise du réel. Il n'y aura pas « une seule école », et ce n'est pas souhaitable, mais il devrait y avoir des écoles pour lesquelles il sera communément clair qu'elles exercent la responsabilité de diffuser, face à " un seul monde », un type totalement spécifique et irremplaçable de message. "Faut-il se plaindre que le monde contemporain manque de repères ou bien ne doit-on pas se dire au contraire que la scolarisation longue, pour la première fois dans l'histoire de l'humanité, de la majorité des jeunes humains crée pour l'école l'immense responsabilité de leur proposer ces repères-mêmes ? " ${ }^{4}$.

4. GAUTHIER, R.-F., (2006), Les contenus de l'enseignement secondaire dans le monde : état des lieux et choix stratégiques, Paris, UNESCO. 
Poser la question des contenus et valeurs qu'enseigne l'école revient, on s'en rend compte, à poser la question même de la fonction présente et future de l'école dans un monde fracturé quant aux valeurs qu'il défend : à cet égard, on peut se demander si les écoles doivent être soumises à tous les jeux politiques et sociaux, ou bien si le "seul monde » n'a pas besoin qu'elles s'en distancient à nouveau, pour mettre à jour quelque conscience planétaire qu'il se joue des choses en large partie communes dans les apprentissages de tous les enfants. Le droit à l'éducation, par exemple, ne devrait-il pas devenir un droit à la qualité, à la probité, à la conformité aux principes généraux du droit, etc., des contenus dispensés dans les écoles?

On voit le paradoxe : on considère comme un positionnement positif que les contenus scolaires ne soient plus sanctuarisés, c'est-à-dire séparés de la société et du monde jusqu'à les oublier, mais on appelle confusément peut-être aussi quelque nouvelle sanctuarisation du monde scolaire, non seulement pour le protéger des passions du monde, mais par la conscience émergente qu'un nouvel ordre de savoirs, compétences et valeurs scolaires, non pas uniformes partout, mais associés et uniformément responsables, est désormais à inventer.

\section{BiBLIOGRAPHIE}

LINDSKOG I. (2009) : "Valeurs et contenus dans le curriculum suédois ». Revue internationale d'éducation de Sèvres. [http://www.ciep.fr/ries/colloque-2009/atelier-D.php].

MULEY D.S. (2009) : «L'éducation fondée sur les valeurs dans les écoles indiennes ». Revue internationale d'éducation de Sèvres. [http://www.ciep.fr/ries/colloque-2009/ atelier-D.php].

PLANEL C. (2009) : "Valeurs et contenus d'enseignement. Le cas de l'Angleterre ». Revue internationale d'éducation de Sèvres. [http://www.ciep.fr/ries/colloque-2009/ atelier-D.php].

RAULIN D. (2009) : "Valeurs et contenus d'enseignement. Le cas français ». Revue internationale d'éducation de Sèvres. [http://www.ciep.fr/ries/colloque-2009/atelier-D. php].

SANÉ A. (2009) : "L'exemple de l'enseignement des sciences et de la technologie au Sénégal. État des lieux, propositions de rénovation et valeurs sous-jacentes ». Revue internationale d'éducation de Sèvres. [http://www.ciep.fr/ries/colloque-2009/atelier-D. php]. 